\title{
Waechter, Matthias: Geschichte Frankreichs im 20. Jahrhundert, 608 S., Beck, München 2019.
}

\author{
Jens Ivo Engels \\ Online publiziert: 7. Mai 2020 \\ (C) Der/die Autor(en) 2020
}

Vorbei sind die Zeiten, in denen vertiefte Kenntnisse französischer Geschichte zum selbstverständlichen Kanon der Ausbildung von Historikerinnen und Historikern hierzulande gehörten. Kaum hat die Frankreichforschung in der Zunft den Stellenwert, der ihr aufgrund der Verflechtungen mit unserem westlichen Nachbarland gebührt.

Umso erfreulicher und nützlicher ist die Gesamtdarstellung der französischen Geschichte des 20. Jahrhunderts aus der Feder des kundigen wissenschaftlichen Grenzgängers Matthias Waechter, der seit vielen Jahren in Südfrankreich lebt, forscht und lehrt. Waechter legt eine konzise und sehr gut lesbare Darstellung auf rund 500 Textseiten vor. Es handelt sich um ein Referenzwerk, vor allem für all jene, die sich für die politische und sozioökonomische Entwicklung im Hexagon interessieren. Bei aller Breite müssen Schwerpunkte gesetzt werden, und diese liegen eindeutig in den genannten Bereichen - allerdings werden immer wieder auch Passagen zu Themen wie Kultur, Alltag, Geschlechterrollen aufgenommen.

Der Aufbau ist chronologisch gestaltet und orientiert sich in Schritten von jeweils gut 20 Jahren an den klassischen Zäsuren. Jeder Abschnitt ist in drei oder vier Kapitel unterteilt, von denen je eines sich einem Panorama der Gesellschaft widmet: „Frankreich um 1900“, ,um 1926“ und so weiter. Diese Aufteilung ermöglicht gute Einblicke in Dynamiken wie auch Strukturen der politischen und gesellschaftlichen Wirklichkeit.

Das 20. Jahrhundert beginnt in den Augen Waechters nicht mit dem Ersten Weltkrieg, sondern mit der Konsolidierung der Dritten Republik um 1880. Es endet nicht im Jahr 1990, vielmehr wird die Darstellung über die Jahrtausendwende fortgesetzt, einige Bemerkungen zielen bis auf die Wahl von Emmanuel Macron, der die Rückkehr zur gaullistischen Staatsauffassung repräsentiere. Waechter verfolgt nicht das Ziel einer großen Erzählung. Es geht also nicht darum, die französische Geschichte der letzten gut hundert Jahre auf einen Nenner oder einen einzigen Grundkonflikt zu reduzieren. So entsteht das breite Panorama eines Landes, das eine Vielzahl von politischen Phasen, Krisen und Konsolidierungen durchlief. Am ehesten noch wird in Waechters Darstellung die Niederlage und Selbstabschaffung der Dritten Repu-

\footnotetext{
J. I. Engels ( $\triangle)$

Technische Universität Darmstadt, Darmstadt, Deutschland

E-Mail: engels@pg.tu-darmstadt.de
} 
blik 1940 als Fluchtpunkt der vorangegangenen Jahrzehnte und als Ausgangspunkt für die folgenden Dekaden konturiert.

Waechters Darstellung zeigt großes Interesse an Ambivalenzen und Gegensatzpaaren. Dazu gehören nicht nur konträre Entwürfe Frankreichs, wie sie beispielsweise die beiden Lager der Dreyfus-Kontroverse um 1900 oder Charles de Gaulle und Philippe Pétain in den frühen 1940er Jahren repräsentierten. Waechter hebt immer wieder auf die im Projekt der République eingeschriebenen Paradoxien ab, wie beispielsweise den unauflöslichen Gegensatz zwischen Gleichheitsversprechen und Unterdrückung im Kolonialismus der ersten Jahrhunderthälfte oder die Widersprüchlichkeit der fortschreitenden Egalisierung in weiten Teilen des Bildungssystems bei immer härterer Abschottung einer oligarchischen Bildungselite in der zweiten Hälfte des Jahrhunderts.

Die Geschichte Frankreichs wird zwar auf der Grundlage der Forschungsliteratur zumal der letzten Jahre geschildert. Wissenschaftliche Kontroversen oder abweichende Interpretationen finden eher sparsame Berücksichtigung (es gibt einige Ausnahmen wie etwa zur Bewertung der Union sacrée von 1914 oder zur Rolle François Mitterrands bei der deutschen Wiedervereinigung).

Es gibt an diesem Buch nur wenig zu kritisieren. Anlass dazu gibt die Neigung des Autors, alle möglichen Ereignisse als besondere Wendepunkte zu beschreiben, obwohl dies die weitere Darstellung nicht rechtfertigt. So werden die Politik der Volksfrontregierung und die soziale Mobilisierung des Jahres 1936 als ,ein drastischer Neuanfang in der Geschichte der französischen Demokratie“ bezeichnet (S. 206), doch wird das Thema kaum wieder aufgenommen und bleibt ein untergeordneter Punkt im Unterkapitel über die Krise der 1930er Jahre. Auch schleichen sich gelegentlich kleine Widersprüche ein: Während einerseits die Dezentralisierung der Mitterrand-Ära als sehr weitreichende Weichenstellung auch in der Kultur bezeichnet wird (S. 440-441), unterstreicht Waechter an anderer Stelle den ,kaum gebrochenen Pariser Zentralismus“ in Bildung und öffentlichem Leben (S. 478) in dieser Zeit. Letztlich handelt es sich lediglich um redaktionelle Ungereimtheiten.

Matthias Waechters Buch bietet eine umfassende, gut lesbare und zuverlässige Darstellung des 20. Jahrhunderts in Frankreich auf der Grundlage des State of the Art. Vor allem Einsteigern in die französische Geschichte sei das Buch ans Herz gelegt.

Funding Open Access funding provided by Projekt DEAL.

Open Access Dieser Artikel wird unter der Creative Commons Namensnennung 4.0 International Lizenz veröffentlicht, welche die Nutzung, Vervielfältigung, Bearbeitung, Verbreitung und Wiedergabe in jeglichem Medium und Format erlaubt, sofern Sie den/die ursprünglichen Autor(en) und die Quelle ordnungsgemäß nennen, einen Link zur Creative Commons Lizenz beifügen und angeben, ob Änderungen vorgenommen wurden.

Die in diesem Artikel enthaltenen Bilder und sonstiges Drittmaterial unterliegen ebenfalls der genannten Creative Commons Lizenz, sofern sich aus der Abbildungslegende nichts anderes ergibt. Sofern das betreffende Material nicht unter der genannten Creative Commons Lizenz steht und die betreffende Handlung nicht nach gesetzlichen Vorschriften erlaubt ist, ist für die oben aufgeführten Weiterverwendungen des Materials die Einwilligung des jeweiligen Rechteinhabers einzuholen.

Weitere Details zur Lizenz entnehmen Sie bitte der Lizenzinformation auf http://creativecommons.org/ licenses/by/4.0/deed.de. 